\title{
Observation participante ou participation observante? Usages et justifications de la notion de participation observante en sciences sociales
}

\section{Soulé Bastien, Ph.D.}

Université de Caen Basse-Normandie

\section{Résumé}

La notion de participation observante est fréquemment mobilisée au sein de la terminologie propre aux enquêtes de terrain. Les justifications du recours à ce terme sont plurielles, oscillant entre simple recherche d'originalité et usages finement argumentés. Après avoir rappelé la diversité des formes d'observation participante, nous procédons dans cet article à un examen des usages de la notion de participation observante, au croisement de cinq justifications méthodologiques. Au-delà de cette diversité, trois cas de figure apparaissent. Certains usages attestent d'une acception rétrécie de la notion d'observation participante; d'autres traduisent une volonté de précision méthodologique, en réaction aux délimitations floues et au caractère très général de la démarche d'observation participante; enfin, la séduction opérée par ce terme alternatif et l'effet de mode semblent constituer un élément explicatif non négligeable.

Mots clés

OBSERVATION PARTICIPANTE, PARTICIPATION OBSERVANTE, METHODOLOGIE

\section{Introduction}

Au sein de la terminologie spécifique aux enquêtes de terrain, la notion de participation observante $(\mathrm{PO})$ semble de plus en plus fréquemment préférée à celle d'observation participante (OP). Cette mobilisation traduit cependant des objectifs fort disparates : souligner un investissement particulièrement prolongé sur le terrain, suggérer la prépondérance de la participation sur l'observation, signaler le passage de la «participation pure» à l'observation par une « conversion à la recherche », etc. Bref, il existe des façons très différentes de justifier le recours à ce terme alternatif, ce qui génère en toute logique quelques 
incompréhensions (Welzer-Lang, 2001): les simples formulations visant l'élégance côtoient en effet des usages beaucoup plus finement argumentés.

Dresser un inventaire des recherches mettant à contribution ce terme se révélerait fastidieux et sans grand intérêt. Par l'intermédiaire de ce court article, nous proposons plus modestement un recensement des justifications méthodologiques mises en avant par les utilisateurs du terme de PO. Loin de prétendre à l'exhaustivité, cet exercice entend couvrir la diversité des arguments auxquels les chercheurs ont recours. Dans un second temps, cet état des lieux permettra d'alimenter une réflexion approfondie sur la pertinence contextuelle de l'appellation en question.

Pouvant a priori passer pour une entreprise normative, cette contribution vise simplement la clarté terminologique, étant entendu que les formes très variées d'OP d'ores et déjà distinguées dans la littérature en sciences sociales semblent suffire à caractériser certaines des démarches aujourd'hui qualifiées de PO.

\section{Les diverses méthodes d'observation participante}

L'OP implique de la part du chercheur une immersion totale dans son terrain, pour tenter d'en saisir toutes les subtilités, au risque de manquer de recul et de perdre en objectivité. L'avantage est cependant clair en termes de production de données : cette méthode permet de vivre la réalité des sujets observés et de pouvoir comprendre certains mécanismes difficilement décryptables pour quiconque demeure en situation d'extériorité. En participant au même titre que les acteurs, le chercheur a un accès privilégié à des informations inaccessibles au moyen d'autres méthodes empiriques.

Selon Platt (1983), c'est vers la fin des années 1930 que l'expression «observation participante» semble faire son entrée dans son acception actuelle, en tant que technique de recherche dans laquelle le sociologue observe une collectivité sociale dont il est lui-même membre. Le chercheur se fait dès lors l'étudiant d'acteurs sociaux, par l'entremise d'une interaction de longue durée avec eux (De Sardan, 2001). Au regard de la diversité des formes d'OP concrètement mises en œuvre, parler d'acception de ce terme au singulier paraît quelque peu réducteur.

\section{Observation participante ouverte ou clandestine?}

L'OP clandestine, également dite « couverte», a ainsi peu en commun avec l'OP « ouverte», transparente et déclarée. Plusieurs sociologues classent d'emblée l'OP comme une méthode permettant d'observer «à couvert », sans que l'organisation ou le groupe « infiltré » ne soit prévenu et n'ait à donner son accord (Abercrombie, Hill et Turner, 2000). Dès ses origines, l'OP a ainsi été 
utilisée comme une véritable méthode d'enquête «souterraine », réalisée non seulement sans consentement, mais de surcroît sans dévoilement de l'intention réelle du chercheur. Il s'agit alors généralement d'observer des groupes sociaux de taille réduite, et de type fermé, comme des organisations au sein desquelles les chercheurs prennent un emploi (Abercrombie et al., 2000). Ce type d'OP présente l'avantage de saisir un certain nombre de processus sociaux dans leur contexte «naturel » (De Sardan, 2001), y compris au sein de configurations sociales traditionnellement rétives à l'analyse extérieure (Diaz, 2005).

Cependant, dans son acception la plus large, le terme d'OP décrit une forme d'observation lors de laquelle le chercheur « annonce la couleur ». Il est alors connu en tant qu'observateur extérieur, de la part des membres de la collectivité au sein de laquelle il évolue : le risque sous-jacent est de générer des changements artificiels de comportement; un avantage non négligeable est de ne pas soulever de problèmes d'ordre éthique, du fait de la décision de «jouer cartes sur table ».

\section{Parts respectives de la participation et de l'observation}

Le degré de participation constitue un autre élément central de différentiation entre les formes d'OP (De Sardan, 2001), ainsi qu'un objet central de débat épistémologique: comment concilier la nécessité méthodologique de l'implication dans la vie d'un groupe avec le recul et la mise en perspective nécessaires au rôle de chercheur (Diaz, 2005)?

Les réserves émises par certains sociologues quant à la pertinence méthodologique de l'OP se centrent sur la contradiction pratique que représente le fait d'être à la fois partie prenante du jeu social et observateur distancié (Bourdieu, 1978). D'un côté, le chercheur qui se distancie voit son objet « lui glisser des mains »; de l'autre, celui qui accepte une participation de près doit gérer les « risques de la subjectivation » (Favret-Saada, 1977). Afin de conférer à cette articulation un caractère aussi peu problématique que possible, il est souvent conseillé de se restreindre à ce qu'Adler et Adler (1987) nomment l'observation participante périphérique, qui diffère de l'OP complète de par son implication plus modérée. L'enjeu consiste à ne pas être «aspiré », voire obnubilé par l'action, ce qui bloquerait toute possibilité d'analyse approfondie et se ferait au détriment de l'abstraction. Hughes (1996) qualifie ainsi d'émancipation la démarche dans laquelle le chercheur trouve « un équilibre subtil entre le détachement et la participation »(Diaz, 2005). Il propose une approche ressemblant fort à un compromis : être participant et observateur à temps partiel, c'est-à-dire participant en public et observateur en privé. Cette prudence méthodique est selon Chapoulie (1984, p.598) «le prix à payer pour rester sociologue dans l'aventure de la participation ». 


\section{Le mode d'entrée sur le terrain}

Enfin, un troisième vecteur de différentiation rentre en considération. L'OP par opportunité désigne le cas lors duquel une «participation pure », par le biais d'une « conversion à la recherche », devient observation. L'immersion est dès lors poussée, du fait du rôle joué au sein du groupe et du temps plein de la présence en son sein. La difficulté consiste alors à s'extraire, dans une certaine mesure, des habituelles façons de faire et de penser, afin de développer une réelle capacité d'analyse. La réduction de la distance à l'objet laisse en effet davantage de place à la subjectivité, notamment parce que le chercheur doit autant, voire davantage participer qu'observer.

Dans toute démarche d'OP, on peut ainsi se demander quelle est la part de la participation, et plus globalement ce que l'on entend par «participation » du chercheur (De Sardan, 2001).

\section{Les usages de la notion de participation observante et ses justifications}

Le recours au terme de PO est de plus en plus constaté, sans que les conditions de cet usage ne soient toujours clarifiées. Face au récent succès de cette notion méthodologique, une analyse en profondeur des spécificités brandies pour justifier cette utilisation s'impose.

La notion de PO apparaît fréquemment comme une sorte de figure de style, ayant vocation à souligner un investissement important, ou particulièrement prolongé, au sein d'un groupe, d'une communauté ou d'une organisation. La recherche d'originalité semble dès lors justifier ce choix terminologique, sans réel autre argument ${ }^{1}$. Pinçon et Pinçon-Charlot (2002) utilisent par exemple ce terme pour relater leur participation à diverses cérémonies ou festivités, en tant qu'invités de la grande bourgeoisie; ne faisant pas partie de celle-ci, ils mettent à profit ces épisodes sur un plan empirique. On perçoit ici à quel point la notion de PO se substitue parfois à celle d'OP; en l'absence de motif explicitement mis en avant, on ne peut que constater l'usage indifférencié des termes d'OP et de PO.

Nous mettons aussi volontairement de côté les justifications s'appuyant sur l'exercice préalable d'une fonction (associative, professionnelle...) sur le terrain où démarre la recherche (cas de l'employé devenu chercheur, par exemple). En effet, bien que Brewer (2000) caractérise la PO (observant participation) comme l'utilisation d'un rôle existant pour engager une recherche dans un environnement familier, ce mode d'entrée sur un terrain constitue le point de départ de nombreuses recherches menées par OP depuis 
des décennies (Becker, 1985; Dalton, 1959). Ce seul élément est donc insuffisamment convaincant pour recourir à une terminologie alternative.

Parmi les auteurs cherchant à être plus explicites sur les raisons du recours au terme de $\mathrm{PO}$, quatre usages différents, se recoupant et s'opposant parfois, sont constatés.

\section{La primauté de l'implication interactionnelle et intersubjective sur la prétention à l'observation objective}

En rappelant les limites de l'OP, dues à l'incompatible nécessité d'être simultanément participant et observateur, Lassiter (2000) se prononce pour l'adoption plus réaliste de la notion de PO. On rejoint ici les critiques élargies adressées ci-dessus à l'égard de l'OP comme outil de recherche. Cependant, les conclusions qui sont tirées de ce constat diffèrent nettement de celles formulées supra.

La participation entraîne inévitablement des relations de proximité, voire une intimité avec les acteurs d'un terrain. L'observation constitue pour sa part une activité «naturelle» de tout participant. Mais dans son acception académique la plus rigoureuse, elle est supposée s'appuyer sur une mise à distance objectivée de ces mêmes relations humaines. Pour faire face à ce paradoxe, Tedlock (1991) affirme que les ethnographes doivent accepter de reconceptualiser l'OP en PO. L'enjeu est de taille : il s'agit de dépasser les apparentes contradictions inhérentes à l'OP, pour embrasser complètement, et honnêtement, les inter-subjectivités du travail de terrain ethnographique. En effet, la théorie veut que pendant l'OP, les ethnographes soient «alternativement émotionnellement engagés, en tant que participants, et froidement observateurs, dépassionnés, des vies des autres. Cette étrange démarche n'est pas seulement émotionnellement déstabilisante, mais également suspecte sur un plan moral, du fait que les ethnographes établissent volontairement des relations humaines intimes, avant de les dépersonnaliser (...). Dans l'observation de la participation, à l'inverse, les ethnographes utilisent leurs compétences sociales quotidiennes simultanément pour expérimenter et observer les interactions, les leurs comme celles des autres, au sein de configurations sociales diverses » (Tedlock, 1992, p.13).

L'approche préconisée n'est pas tant destinée à résoudre une difficulté plus ou moins incontournable, qu'à accepter les problèmes, tout comme la richesse, que représente l'intrication du chercheur et de son terrain. L'accent passe de l'observation de l'autre à l'observation de la relation humaine entre soi, en tant qu'ethnographe, et l'autre (les gens avec lesquels l'ethnographe travaille pour produire son étude). L'ethnographe ne peut en effet guère prétendre être objectif et observateur participant : l'ethnographie est définie et 
façonnée par les relations humaines, elle est construction d'une fiction rationnelle, et non-recherche objective de connaissance.

Dès lors, comme l'affirme Emerson (2003, p.410), « la solution est davantage du côté de la prise de conscience des effets de l'enquête que de la tentative de les minimiser (...) On tient pour allant de soi que l'observateur altère ce qu'il observe ${ }^{2}$, mais que ces altérations font partie de l'objet d'étude (...) Le travail de terrain est donc nécessairement de nature interactionnelle et la présence de l'enquêteur a des conséquences dans la vie des enquêtés. Les solutions à la réactivité ne sont pas dans la régularisation, la restriction ou la suppression des interactions sur le terrain. Elles réclament que l'on devienne sensible et réceptif à la façon dont les protagonistes se perçoivent et se traitent les uns les autres. Le chercheur est une source de résultats, non pas de contamination de ceux-ci ».

C'est cette prise de conscience qui appellerait un changement de conception de l'observation, prenant pleinement en considération la participation active du chercheur à son terrain, et en faisant un réel outil de connaissance.

\section{Les cas de conversion expérientielle à un terrain ou un rôle singuliers}

Dans ce cas de figure, c'est l'implication conséquente sur un terrain par certains aspects exotiques qui justifie le recours à la notion de PO. L'objet de cette présence est de comprendre de l'intérieur un phénomène a priori inconnu, en passant du statut initial d'outsider à celui d'insider.

Pfadenhauer (2005) énumère plusieurs aspects par lesquels la PO diffère de l'OP: 1) idéalement, la recherche vise la production de données d'observation, mais aussi de données expérientielles; 2) si des arbitrages sont nécessaires sur le terrain, la participation est prioritaire par rapport à l'observation; 3) le chercheur est centré sur la connaissance de la perspective existentielle de l'insider, identifiée à travers une expérience subjective, plutôt que sur le développement d'un point de vue approximatif d'outsider distant. Pour toutes ces raisons, l'interprétation des données obtenues et des expériences subjectives vécues appelle une analyse d'essence phénoménologique.

En sociologie des religions, la PO implique la participation du chercheur au contexte expérientiel des pratiquants, particulièrement à même de lui permettre d'identifier en connaissance de cause les efforts individuels entrepris pour donner du sens à la vie (Ramstedt, 2002). Pye (2000) différentie ainsi le rôle relativement passif de l'observateur participant, et celui, davantage actif, de participant observateur. Il cite l'exemple de Schott (1997), passé du premier au second lorsque, suivant les instructions d'un devin, il a procédé à un 
sacrifice animal en l'honneur de ses ancêtres. Cet épisode étant censé ouvrir des possibilités élargies de compréhension de ce type d'activités sociales.

Dans un registre tout à fait différent, Wacquant (2000) s'est intéressé aux jeunes du ghetto noir de Chicago fréquentant une salle de boxe. Souhaitant initialement comprendre leurs trajectoires sociales, et leurs éventuelles stratégies de sortie du ghetto, il entendait a priori se limiter à observer et réaliser des entretiens. Cette intention méthodologique s'est d'emblée transformée en OP : «il était impensable de rester assis sur une chaise, d'observer et de parler aux gens en arrivant comme ça, de nulle part (...) du coup, quand le vieux coach m'a demandé "Bon, qu'est-ce que tu veux faire?", j'ai répondu "Et bien, euh... Je voudrais apprendre à boxer", ce qui n'était pas du tout mon intention. »

L'utilisation du terme PO s'est imposée ultérieurement à Wacquant, qui la justifie par le fait qu'il a donné de sa personne, au sens propre du terme, sur le ring comme lors des longues séances d'entraînement, allant jusqu'à envisager de mettre un terme a sa carrière universitaire alors en germe. "Trois ans durant, j'ai participé aux entraînements aux côtés des boxeurs du cru, amateurs et professionnels, à raison de trois à six séances par semaine. À ma propre surprise, je me suis pris au jeu, au point de passer mes après-midi au gym avant de passer entre les cordes disputer un combat officiel ». Soumettre sa chair aux aléas et aux conditionnements de l'univers considéré faisait partie, pour Wacquant, de la compréhension de ce que c'est que «devenir boxeur». C'est une méthode radicalisant d'une certaine manière la théorie bourdieusienne de l'habitus : fabriquer et subir soi-même l'habitus de boxeur pour mieux comprendre l'univers pugilistique.

En endossant le costume de boucher, Blondeau (2002) était décidée à comprendre en détail ce métier. Elle évoque les tensions, relativement habituelles en ethnographie, entre son rôle de chercheur et son implication concrète de terrain. Privilégiant finalement délibérément la participation à l'observation, elle affirme avoir développé dans l'action une mémoire spécifique, plus riche que celle inscrite sur carnet ou bande magnétique. Les données obtenues par participation se révèlent centrales dans la construction du savoir ethnographique. "Si l'immersion dans un terrain est désignée communément en ethnologie comme l'observation participante, je préfère qualifier ma démarche comme une participation observèrent, car c'est avec le tablier de bouchère que j'ai le plus appris et le mieux compris le monde boucher. En affrontant visuellement, tactilement, olfactivement et auditivement les lieux et les pratiques des bouchers, le monde de la boucherie devient sensible, tangible. Un monde avec ses odeurs fortement perceptibles, 
évocatrices et persistantes, ses sonorités de scies à os, ses visions frappantes du jaillissement du sang et des entrailles qui déclenchent des sensations inimaginables. Ce monde éveille des émotions et des représentations profondément intériorisées auxquelles il s'agit de se confronter dans le processus de construction de l'objet, pour comprendre, soi et les autres » (Blondeau, 2002).

À travers ces cas de figure, la participation revêt un caractère singulier et particulièrement marquant, car elle concerne des activités extraordinaires, fort éloignées de l'univers culturel des scientifiques, réclamant un investissement d'autant plus conséquent et coûteux. Ces éléments semblent explicatifs de la volonté de ne pas «simplement» qualifier la démarche d'OP. La PO signifie dès lors que le chercheur fréquente le terrain examiné aussi intensément que possible (Pfadenhauer, 2005). En cas de succès, la qualité des données obtenues est difficilement égalable par d'autres moyens méthodologiques : on recueille en effet des informations à propos de ce que quelqu'un expérimente véritablement du monde, et sur la manière dont est vécue cette expérience. La présence sur le terrain n'est pas seulement destinée à décrypter un système social de l'intérieur; elle vise à comprendre ce que c'est qu'être un acteur de ce terrain, compréhension qui demeure toutefois ancrée dans une expérience essentiellement subjective.

\section{Une participation intense éclipsant momentanément la lucidité et la disponibilité intellectuelle du chercheur}

Comme Blondeau (2002) l'évoque supra, le quotidien de participant prend parfois le dessus, empêchant alors de se comporter comme un «vrai chercheur», disposant de temps pour noter des informations, discuter, et prendre du recul vis-à-vis de son objet. C'est la troisième voie identifiée dans la littérature en sciences sociales pour justifier le recours au terme de PO. Dès 1958, Gold avait établi une distinction fondamentale entre l'observateur complet, l'observateur en tant que participant, le participant en tant qu'observateur et le participant complet, catégorisant ainsi graduellement des modes de présence sur le terrain allant de l'implication minimum à l'implication maximum.

Lors d'une OP, la personne «se mêle au milieu observé, mais peut en sortir à chaque instant, et ne se trouve donc jamais exactement dans la position sociale, ni dans la disposition psychologique des acteurs étudiés » (Damon, 2002, p.20); à l'inverse, dans la PO, la personne, qui fait partie du milieu, prend à certains moments du recul pour l'observer (Marpsat, 2005). L'expérience relatée par Rocher (1984) cadre avec cette description : " après quelques 25 ans de vie universitaire ininterrompue, je quittais pour la première 
fois l'université, d'une manière provisoire il est vrai, pour accepter un poste à temps complet dans la fonction publique québécoise. Sous-ministre du ministre d'État au Développement culturel, c'est de très près cette fois-ci que je participai à l'exercice du pouvoir politique (...) Pendant deux années et demie, j'ai donc pratiqué ce que l'on appelle dans notre langage l'observation participante, ou peut-être était-ce plus exactement de la participation observante, car le rythme et les exigences du travail quotidien ne m'ont pas permis de prendre toutes les notes que j'aurais voulu et de tenir un véritable journal d'observateur, comme je l'avais souhaité au départ (Rocher, 1984). La PO constitue dans cette optique une forme plus ou moins appauvrie d'OP, sur le plan de l'analyse réalisée. C'est loin d'être toujours le cas.

La manière dont se préparent et se déroulent concrètement les expéditions polaires, sur le plan logistique, ainsi qu'en situation effective, c'est-à-dire face au vent, au froid, à la fatigue et aux attaques d'ours, a été analysée par Lièvre et Rix (2005). La différence fondamentale entre observation participante et participation observante réside à leurs yeux dans l'activité principale du chercheur: s'il est avant tout acteur de terrain, se transformant uniquement en chercheur une fois sa mission terminée (par la prise de notes, par exemple), on peut parler de participation observante. «Quand vient le moment de démonter la tente, il doit participer à cette action comme les autres; ensuite seulement, il pourra prendre des notes. Ceci suppose qu'il soit un praticien expérimenté des expéditions polaires à ski, car s'il est déjà débordé par ce qu'il a à faire en tant qu'acteur, il ne pourra pas mener à bien sa recherche » (Lièvre et Rix, 2005, p.6). Si, au contraire, la priorité va à l'observation, avec des prises d'initiative plus rares de la part de quelqu'un qui n'est pas familier du terrain, il est alors question d'observation participante, consistant éventuellement en une forme d'apprentissage. "Le chercheur doit être capable de suivre l'expédition (...) mais il n'a pas un statut d'expert dans l'équipe; son discours est plutôt: dis-moi ce que tu fais; apprends-moi comment on réalise cette opération; moi, je ne sais pas comment on s'y prend » (Lièvre et Rix, 2005, p.6).

Au-delà de la diversité des usages mise à jour, l'expression PO sert dans tous les cas présentés à souligner la priorité accordée, au quotidien, à la participation. La qualité du recueil des données pâtit éventuellement de la mise au second plan de l'observation, puisque l'analyse ne se développe que dans les rares interstices de temps qui lui sont favorables. Sur certains terrains nécessitant une forte implication personnelle, la priorité accordée à la participation constitue la condition sine qua non de la réalisation d'une observation. 


\section{Une participation intellectuellement engagée}

Dans ce dernier cas de figure, un engagement intellectuel (celui du militant) ou une visée appliquée (perspective de recherche-action) font du chercheur un acteur central du changement opéré et/ou espéré. C'est le cas du récit rapporté par Verrier (2006), alors conducteur de trains à la $\mathrm{SNCF}$, sur sa participation au mouvement de grève de novembre 1995.

«Sur le terrain, j'étais davantage dans la participation parce que l'observation passait au second plan, elle n'était plus alors mon objectif premier. Par l'intermédiaire de l'écriture, j'essayais cependant de retrouver une certaine forme de distanciation, de rétablir des dimensions d'observation réflexive. J'étais pris dans la grève, entraîné et impliqué par elle, peut-être malgré moi en certaines circonstances, mais je tentais aussi d'en être un acteur délibérément engagé, avec mes caractéristiques individuelles et émotionnelles. Chaque soir ou chaque nuit, éloigné du terrain de la grève, la pré-écriture du texte offrait l'occasion d'une prise de recul par rapport à l'action et à l'implication, et permettait certainement de mieux s'y inscrire le lendemain tout en se livrant à des retours sur soi. De fait naissait une sorte de dichotomie, je me retrouvais tiraillé entre un besoin d'action sur le terrain et une posture de réflexion et de travail intellectuel sur la grève. Il me semble cependant que cela débouchait sur un enrichissement réciproque entre d'une part l'observation et l'action et d'autre part la symbolisation de cette action » (Verrier, 2006).

Ayant à cœur la réalisation d'un projet dépassant l'observation scientifique d'un objet ou d'un terrain, l'observateur ne saurait dans de pareilles circonstances être cet analyste froid, uniquement centré sur sa fonction de chercheur, qui n'est du reste a priori pas principale : il est avant tout acteur d'un événement et/ou partie prenante d'un processus en cours.

\section{Conclusion}

La souplesse de la notion d'observation participante, matérialisée par les formes extrêmement diverses que peut revêtir sa mise en œuvre, constitue un terrain propice aux variations terminologiques ${ }^{3}: l^{\prime} \mathrm{OP}$ peut ainsi être ouverte ou clandestine, périphérique ou complète, entriste ou opportuniste; le chercheur, pour sa part, s'apparente plutôt à l'observateur en tant que participant, ou au participant en tant qu'observateur, parfois après avoir été «participant pur ». $\mathrm{Au}$ regard de ces multiples configurations, le recours au terme de PO semble parfois résulter d'une acception rétrécie de la notion d'OP.

Dans l'ensemble, les auteurs recourant à la formule alternative de PO cherchent à caractériser un rapport spécifique au terrain (conversion expérientielle, participation ou engagement particulièrement intense, etc.). Des zones de recouvrement ne manquent pas d'apparaître entre certaines 
justifications méthodologiques évoquées supra. À tel point qu'un dénominateur commun à toutes les approches émerge : pour diverses raisons, la priorité va à la participation au terrain investigué, prépondérante vis-à-vis de l'observation. Très fortement impliqués sur leur terrain, voire enchâssés dans celui-ci, les chercheurs recourant au terme de PO revendiquent un rapport singulier au terrain, qui les place en décalage avec «la bonne pratique méthodologique » relatée dans les manuels : observer et participer à parts égales, en veillant à ne pas sacrifier l'un au bénéfice de l'autre ${ }^{4}$.

Ce jeu sur la formulation ne consiste pas seulement en une inversion des termes; l'adjectivation de l'un et la substantivation de l'autre confortent la position exprimée par Favret-Saada (1990), qui qualifie 1'OP d'oxymore. Audelà, l'ethnographe dénonce l'objectivité illusoire vers laquelle est censé tendre l'observateur distant. «De tous les pièges qui menacent notre travail, il en est deux dont nous avions appris à nous méfier comme de la peste : accepter de participer au discours indigène, succomber aux tentations de la subjectivité. Non seulement il m'a été impossible de les éviter, mais c'est par leur moyen que j'ai élaboré l'essentiel de mon ethnographie ». L'enjeu consiste précisément, selon Favret-Saada (1990), à « être affecté » par le terrain d'étude, condition sine qua none de l'observation participante ${ }^{5}$. Pour la plupart, les adeptes de la PO se rapprochent de cette manière d'envisager la relation au terrain.

L'accent a été mis dans cet article sur les raisons avancées pour justifier le recours au terme de PO. Les cas d'usages n'étant accompagnés d'aucune précision méthodologique semblent toutefois fréquents. Il existe ainsi au sein de la terminologie spécifique aux enquêtes de terrain des termes ad hoc, parfois peu éprouvés, mais dont l'usage s'impose malgré tout au sein de la communauté scientifique. Cette simplicité d'emprunt autorise parfois le contournement de l'exercice de définition, mais en contrepartie, les limites conceptuelles ou méthodologiques de ces objets sont fréquemment outrepassées, quand bien même l'inconsistance guette (Passeron, 2006).

\section{Notes}

${ }^{1}$ Une simple recherche par mot clé sur Internet permet d'observer la façon anecdotique dont nombre de chercheurs en sciences sociales mobilisent ce terme: "Cette observation stricte prend parfois des tournures d'observation participante et même de participation observante... »; "A cette longue expérience de recherche correspond un travail de terrain en profondeur, qui a essentiellement consisté en la pratique de participation observante et d'observation participante... »; "Il convient au mode de l'observation-participante, ici même participation-observante, et au caractère saisissant du phénomène... »; "Cette méthode qui, en détournant la formule classique, 
peut être qualifiée de participation observante...»; "Observation participante (et participation observante)... »; «OP... Ou ne devrait-on pas plutôt dire PO ?»

${ }^{2}$ Plusieurs interprétations peuvent être faites de ce constat relativement classique: l'altération peut tout d'abord concerner les acteurs et relations étudiés, qui seront plus ou moins affectés, à plus ou moins long terme, par la présence temporaire de l'observateur (éventuellement après le départ de celui-ci); par ailleurs, cette présence peut exercer une influence sur les comportements observés, qui ne sont plus dès lors ceux, «naturels », que souhaiterait appréhender le chercheur in situ; enfin, dans une perspective constructiviste, l'altération peut traduire les distorsions que ne manque pas d'imprimer le chercheur à son terrain, à travers la compréhension singulière qu'il en développe.

${ }^{3}$ D'autres termes, comme celui d'immersion (utilisé à plusieurs reprises dans ce texte), correspondent à ce que plusieurs chercheurs décrivent comme une PO : expérience forte, absorbante, monopolisant toute l'attention de l'observateur.

4 Deux logiques potentiellement contradictoires sont en jeu (Schinz, 2002): l'observation que requiert l'appartenance au monde académique, et la participation qu'ordonne le terrain. "Dès lors, durant la période de présence de l'ethnographe sur son terrain, on peut assister à une sorte de combat entre ces deux logiques, entre deux systèmes de valeurs conflictuels (mais pas nécessairement très conflictuels...) »

${ }^{5}$ Lors de son enquête sur la sorcellerie, Favret-Saada a pu percer à jour des croyances au moyen d'une «chute dans la déraison» qui a pris la forme d'une «entrée en sorcellerie »: d'ethnographe, elle est temporairement devenue actrice du système sorcier (« désorceleuse », puis ensorcelée).

\section{Références}

Abercrombie, N., Hill, S. \& Turner, B.S. (2000). Participant Observation. Dans Dictionary of Sociology ( $4^{\text {th }}$ ed.) (p.256). New York : Penguin Books. Adler, P. \& Adler, P. (1987). Membership roles in field research. Newbury Park : Sage.

Becker, H.S. (1985). Outsiders : études de sociologie de la déviance. Paris: Métailié.

Blondeau, C. (2002). La boucherie: un lieu d'innocence ? http://www.ethnographiques.org/2002/Blondeau.html (Page consultée le 11 mars 2006).

Bourdieu, P. (1978). Sur l'objectivation participante. Réponse à quelques objections. Actes de la recherche en sciences sociales, 23, 67-69.

Brewer, J.D. (2000). Ethnography. Buckingham : Open University Press.

Chapoulie, J.M. (1984). Everett C. Hughes et le développement du travail de terrain en sociologie. Revue française de sociologie, 25, 582-608.

Dalton, M. (1959). Men who manage. New York : Wiley.

Damon, J. (2002). La question SDF. Critique d'une action publique. Paris: PUF. 
De Sardan, J.P.O. (2001). L'enquête de terrain socio-anthropologique, Enquête, $8,63-81$.

Diaz, F. (2005). L'observation participante comme outil de compréhension du champ de la sécurité. http://champpenal.revues.org/document79.html (Page consultée le 12 février 2006).

Emerson, R. (2003). Le travail de terrain comme activité d'observation. Perspectives ethnométhodologistes et interactionnistes. Dans D. Céfaï (Éd.) L'enquête de terrain. Paris : La Découverte/MAUSS.

Favret-Saada, J. (1977). Les mots, la mort, les sorts. Paris : Gallimard.

Favret-Saada, J. (1990). Etre affecté, Gradhiva, 8, 3-10.

Gold, R.L. (1958). Roles in sociological field observations. Social Forces, 36, 217-223.

Hughes, E.C. (1996). Le regard sociologique : essais choisis (textes rassemblés et présentés par Jean-Michel Chapoulie). Paris : Edition de l'EHESS.

Lassiter, L.E. (2000). Authoritative Texts, Collaborative Ethnography, and

Native American Studies. The American Indian Quarterly, 24, 601-614.

Lièvre, P. \& Rix, G. (2005). Le management des expéditions polaires. Revue Française de Comptabilité, 383, 2-8.

Marpsat, M. (2005). Associer les méthodes quantitatives et qualitatives : l'étude du journal en ligne d'Albert Vanderburg, cybernaute et sansdomicile. XXVe Congrès International de la Population, organisé par l'Union Internationale pour l'Etude Scientifique de la Population, Tours, 18-23 Juillet.

Passeron, J.-C. (2006). Le raisonnement sociologique. Paris : Albin Michel.

Pfadenhauer, M. (2005). Ethnography of Scenes. Towards a Sociological Lifeworld Analysis of (Post-traditional) Community-building. Forum : Qualitative Social Research, 6, [En ligne]. http://www.qualitativeresearch.net/fqs-texte/3-05/05-3-43-e.html (Page consultée le 17 avril 2006).

Pinçon, M. \& Pinçon-Charlot, M. (2002). Voyage en grande bourgeoisie. Journal d'enquête. Paris : PUF.

Platt, J. (1983). The Development of the « Participant Observation» Method in Sociology: Origin Myth and History. Journal of the History of the Behavioral Sciences, 19, 379-393.

Pye, M. (2000). Review of Rüdiger Schott's « Orakel und Opferkulte bei Völkern der westafrikanischen Savanne ». Marburg Journal of Religion, 5, 27-29.

Ramstedt, M. (2002). Empowering the Self. The Authority of Transformative Experience and New Forms of Religiosity in Secularized Dutch Society. 
The 2002 CESNUR International Conference (Minority Religions, Social Change, and Freedom of Conscience), Salt Lake City \& Provo (Utah), June 20-23.

Rocher, G. (1984). Le sociologue et le pouvoir ou comment se mêler des affaires des autres. Dans Lévesque et al. (Dirs) Continuité et rupture. Les sciences sociales au Québec (Tome 2) (p. 369-383). Montréal : Presses de l'Université de Montréal.

Schinz, O. (2002). Pourquoi les ethnologues s'établissent en enfer? http://www.ethnographiques.org/2002/Schinz.html (Page consultée le 18 octobre 2007).

Schott, R. (1997). Orakel und Opferkulte bei Völkern der westafrikanischen Savanne. Opladen : Westdeutscher Verlag.

Tedlock, B. (1991). From Participant Observation to the Observation of Participation: The Emergence of Narrative Ethnography. Journal of Anthropological Research, 47, 59-94.

Tedlock, B. (1992). The Beautiful and the Dangerous : Dialogues with the Zuni Indians. New York : Viking.

Verrier, C. (2006). Journal de grève à la Gare du Nord, [En ligne]. http://www.barbier-rd.nom.fr/journal.greve.html (Page consultée le 12 mai 2006).

Wacquant, L.J.D. (2000). Corps et âme: carnets ethnographiques d'un apprenti boxeur. Marseille: Agone.

Welzer-Lang, D. (2001). L'échangisme: une multisexualité commerciale à forte domination masculine. Sociétés Contemporaines, 41/42, 111-131.

Bastien Soulé est sociologue, maître de conférences à l'Université de Caen BasseNormandie. Il est chercheur au Centre de Recherche sur les Activités Physiques et Sportives. Ses recherches portent notamment sur l'engagement corporel et sur la gouvernance du sport. 